Illinois State University

ISU ReD: Research and eData

Theses and Dissertations

$4-2-2020$

\title{
Physical Therapists Perceptions Of Patient Adherence And Strategies For Facilitating Motivation
}

Teadora Markovski

Illinois State University, tmarkovski3@gmail.com

Follow this and additional works at: https://ir.library.illinoisstate.edu/etd

Part of the Kinesiology Commons, and the Psychology Commons

\section{Recommended Citation}

Markovski, Teadora, "Physical Therapists Perceptions Of Patient Adherence And Strategies For Facilitating Motivation" (2020). Theses and Dissertations. 1243.

https://ir.library.illinoisstate.edu/etd/1243

This Thesis is brought to you for free and open access by ISU ReD: Research and eData. It has been accepted for inclusion in Theses and Dissertations by an authorized administrator of ISU ReD: Research and eData. For more information, please contact ISUReD@ilstu.edu. 


\section{PHYSICAL THERAPISTS PERCEPTIONS OF PATIENT ADHERENCE AND STRATEGIES FOR FACILITATING MOTIVATION}

\section{TEADORA MARKOVSKI}

49 Pages

Physical therapists (PTs) are professionally required to undertake a comprehensive examination of their patients and provide consultation within their expertise, yet also have the task to motivate patients to adhere to therapy. Self-determined motivation, as a positive predictor of treatment adherence, can be facilitated by promoting patient autonomy, competence and relatedness. However, a greater understanding is needed of the context specific strategies PT's can use to facilitate motivation. The purpose of this study was to explore physical therapists' perceptions of the factors affecting patient adherence and motivation, and the strategies and methods to facilitate patient adherence and motivation. Thirteen physical therapists from around Illinois were contacted through purposive and convenience sampling to participate in semistructured interviews pertaining to their perceptions of patient adherence and motivation throughout physical therapy. Using a critical realist philosophical perspective, the semistructured interviews followed an interview guide focused on physical therapists' experiences with patient motivation and adherence. Participants included eight Female and five Male physical therapists with an average of 5.7 years experiences in the profession. After the completion of the interviews, a theoretical thematic analysis was conducted to identify themes related to individual and situational factors influencing motivation, and motivation strategies related to satisfying the basic needs theory and the importance of autonomy, competence, and 
relatedness to patient motivation. Findings show physical therapists believed patients individual factors (e.g., expectations) and environmental factors (e.g., clinic environment) both influence adherence and motivation; and that promoting patient buy-in (autonomy), building rapport with patients (relatedness), and enhancing self-recognition of progress (competence) can help patients become more adherent to physical therapy. Physical therapists believed they play a role in facilitating patient adherence and motivation, but the role is dependent on the patient's mindset, knowledge, and expectations of physical therapy. Findings are presented to highlight the unique insights gained from the study along with practical recommendations for PT's practices and strategies to motivate patients.

KEYWORDS: physical therapy; motivation; adherence 


\section{PHYSICAL THERAPISTS PERCEPTIONS OF PATIENT ADHERENCE AND STRATEGIES \\ FOR FACILITATING MOTIVATION}

TEADORA MARKOVSKI

A Thesis Submitted in Partial Fulfillment of the Requirements for the Degree of

MASTER OF SCIENCE

School of Kinesiology and Recreation

ILLINOIS STATE UNIVERSITY 
(C) 2020 Teadora Markovski 


\title{
PHYSICAL THERAPISTS PERCEPTIONS OF PATIENT ADHERENCE AND STRATEGIES FOR FACILITATING MOTIVATION
}

\author{
TEADORA MARKOVSKI
}

COMMITTEE MEMBERS:

Scott Pierce, Chair

Anthony Amorose 


\section{ACKNOWLEDGMENTS}

I would like to recognize those who played a role in assisting with the completion of this project. My gratitude first goes to my committee chair, Scott Pierce, for his mentorship and guidance throughout the entirety of this project. Additionally, I would like to recognize my committee, Tony Amorose, for his support and encouragement as well. Without their guidance and help this thesis would not have been possible. I would also like to thank my friends and family for their never-ending support and understanding throughout the process. Your encouragement and belief in my ability led to success and completion of this project.

T. M. 


\section{CONTENTS}

Page

ACKNOWLEDGMENTS

CHAPTER I: INTRODUCTION 1

What is Physical Therapy? 2

$\begin{array}{ll}\text { Patient Adherence } & 2\end{array}$

Patient Motivation and its Influence on Patient Adherence $\quad 6$

The Importance of the Therapist and Patient Relationships 9

Purpose of the Study 11

CHAPTER II: METHODS 12

$\begin{array}{ll}\text { Research Approach } & 12\end{array}$

Sampling Approach and Participants $\quad 12$

$\begin{array}{ll}\text { Procedures } & 13\end{array}$

$\begin{array}{ll}\text { Semi-Structured Interviews } & 14\end{array}$

$\begin{array}{ll}\text { Data Analysis } & 15\end{array}$

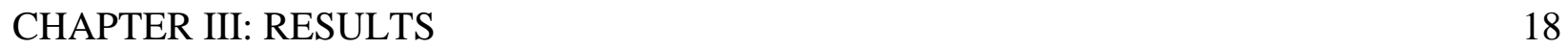

Physical Therapists Perceptions of Factors Influencing Patient Motivation 18

Individual Factors Influencing Patient Adherence and Motivation 19

Environmental Factors Influencing Patient Adherence and Motivation $\quad 24$

Physical Therapists Perceptions of the Strategies and Methods to Facilitate

$\begin{array}{ll}\text { Adherence and Motivation } & 28\end{array}$

$\begin{array}{ll}\text { Autonomy } & 29\end{array}$

$\begin{array}{ll}\text { Competence } & 31\end{array}$ 
$\begin{array}{ll}\text { Practical Recommendations } & 40\end{array}$

Limitations and Future Research $\quad 42$

$\begin{array}{ll}\text { Conclusion } & 43\end{array}$

$\begin{array}{ll}\text { REFERENCES } & 44\end{array}$

APPENDIX A: INTERVIEW GUIDE 49 


\section{CHAPTER I: INTRODUCTION}

Physical Therapists (PTs) play a key role in helping people return from an injury to activities they were previously a part of. This is done through sessions occurring multiples times a week for weeks or months until a patient has reached the goals they have set or have been set based on a rehab protocol. A patient may express their goals and motivations to follow through with PT but end up doing the opposite which leads to non-adherence of their program. Studies have been done to identify why patients choose to continue with a rehabilitation program or not (Babatunde et al., 2017; Enyon et al., 2017; Jack et al., 2010), highlighting the focus on patient adherence. Adherence has been defined as “the extent to which a person's behavior corresponds with agreed recommendations from a healthcare provider" (WHO, 2003), and is clinically determined by the number of appointments attended, meeting goals, performing exercises at home and any other factors that affect a patient following through with therapy (McLean, Burton, Bradley \& Littlewood, 2010). In addition to considering what the patient is doing, it is also important to consider the role of the PT-patient relationship in adherence. The relationship that a PT and patient create is unique since it doesn't reflect relationships that patients have with other health professionals. PTs can help create an environment that patients need to succeed and adhere to a rehabilitation program. The relationship between PT and patient can help foster motivation, but it is also important to consider the steps that need to be taken for the patient to create their own motivation. Considering how patient motivation and adherence work within physical therapy can help clinicians and patients create a better environment for people to feel successful in treatment. The purposes of this study were to explore PT's perceptions of: (1) the factors affecting patient adherence and motivation; and (2) the strategies and methods to facilitate patient adherence and motivation. 


\section{What is Physical Therapy?}

Physical therapy is provided by physical therapists (PTs) to individuals and populations to develop, maintain, and restore maximum movement and functional ability throughout the lifespan (World Confederation of Physical Therapy, 2016). PTs are qualified and professional required to undertake a comprehensive examination/assessment of the patient/client and provide therapy under circumstances where movement and function are threatened by aging, injury, pain, diseases, disorders, conditions or environment factors. During assessment, PTs evaluate findings from their assessment, which based on functional tests patients are asked to perform, to make clinical judgements and formulate a plan of care regarding the patient. Once a plan of care is established, the PT will implement an intervention/treatment program. PTs are able to determine, from these visits, how patients respond and progress to their plan of care, allowing PTs to make necessary adjustments as needed depending on the patient's progress and ability to follow selfmanagement strategies, such as home exercise programs.

\section{Patient Adherence}

One major objective for a PT is to support and promote patient adherence and motivation. Adherence is defined as "the extent to which a person's behavior corresponds with agreed recommendations from a healthcare provider" (WHO, 2003). In Physical therapy, adherence is related to attendance of appointments, following advice, undertaking prescribed exercises, frequency of undertaking prescribed exercise, correct performance of exercises or doing more or less than advised (Jack, McLean, Moffett, \& Gardiner, 2010).

To understand the nature of patient adherence in the physical therapy process, Jack and colleagues (2010), conducted a systematic review to identify barriers that individuals have when adhering to musculoskeletal outpatient treatment and what clinicians can do to overcome barriers 
that are present. Twenty studies were selected for the review and identified evidence of the following barriers to treatment adherence: low levels of physical activity at baseline or in previous weeks, low self-efficacy, depression, anxiety, helplessness, poor social support or anxiety, greater perceived number of barriers to exercise, and increased pain levels during exercise. Poor self-efficacy was found to affect an individual's confidence and was identified as a barrier to treatment adherence. Additionally, it was noted that there are still some barriers such as transportation and socio-economic status that haven't been studied thoroughly (Tuakli-Wosornu, Selzer, Losina, \& Katz, 2016).

Furthermore, research has been conducted in outpatient settings regarding patient exercise compliance in physical therapy considered by patient and physical therapist factors (Sluijs, Kok \& van der Zee, 1993). Patients were asked questions about different aspects of exercise compliance/adherence in physical therapy. The researchers considered barriers perceived by patients, perceived helplessness and lack of positive feedback. Main barriers patients brought up were lack of motivation and lack of time to perform exercises expected of them. It was shown that patients who were motivated to overcome an injury were more likely to be compliant than their non-compliant counterparts (Sluijs et al., 1993). Similar results were found in a study by Brewer and colleagues (2003) when considering age differences when trying to predict adherence. The participants in this study were assessed using questionnaires regarding self-motivation, social support, athletic identity, and psychological distress before anterior cruciate ligament (ACL) reconstruction. After surgery, patients recorded their completion of home exercise programs and their therapists recorded attendance at, and adherence during, physical therapy sessions. While self-motivation was perceived to be important to all ages in the 
study, it was more prominent in older participants versus the adolescents and young adults in the study (Brewer et al., 2003).

Barriers to treatment adherence are particularly important to consider in physical therapy settings to understand how a PT facilitates patient adherence. McLean and colleagues (2010) considered intervention options to increase adherence in physical therapy settings. Five studies focused on self-report assessments of adherence to exercise programs, collecting minutes of physical activity, amount of appointments attended and others (McLean et al., 2010). It is important to take into consideration both clinician and patient centered assessments of adherence when it comes to their treatment plan. The clinician centered questionnaires that were mentioned assess perception of individuals engagement in inpatient setting (HERES), rate patient's participation during a session (PRPS), and rate the degree in which a patient exerts themselves (SIRAS) (McLean et al., 2017). The patient centered questionnaires include questions that assess exercise adherence in older patients (AESOP), measure physical activity as a measure of exercise adherence in daily life (CHAMPS), evaluate exercise in injured athletes (RAQ-M), and measuring the tendency for an athlete to be overly adherent to rehab (ROAQ) (McLean et al., 2017). The patient and clinician centered assessments consider different aspects of the physical therapy process and don't always line up with one another. Using assessments and questionnaires was viewed to be important to help PT's understand how and why patients adhere to therapy programs, and then provide explicit strategies to facilitate adherence.

Understanding these barriers to exercise compliance in physical therapy can help PTs cater physical therapy programs to include overcoming patient barriers. Providing patients with explicit verbal instructions and using motivational techniques can aide individuals in becoming more active. Techniques such as positive feedback, rewards, written goals, and exercise 
diaries/journals can be helpful for individuals when keeping track of PA levels (Jack et al., 2010). In a study done by Peek and colleagues, they wanted to identify perceptions that physiotherapists had about patient adherence. They found that some patient characteristics that PTs identified as positive factors to patient adherence included self-motivation, self-confidence, belief the exercises will help and willingness to exercise. Ninety-eight PTs out of the 298 included in the study stated that these characteristics portrayed adherence in their patients (Peek, Carey, Sanson-Fisher, \& Mackenzie, 2017).

As the most recent review of literature on patient adherence, Goddard, Roberts, ByronDaniel, and Woodford (2020) recently conducted a systematic review of the psychological factors involved in adherence for injury rehabilitation. While this review focused solely on athletes and sport-related injuries, the findings and conclusions provide unique insights to understanding patient adherence. Specifically, the review of 17 research studies found that adherence was determined by factors from two main categories: individual/person and environmental/situational. Individual-specific factors included the impact of the injury, justification for adherence, motivation, self-efficacy, coping, social support, locus of control, cognitive appraisal, and psychological skills. Environmental factors included characteristics, strategies and effectiveness of the therapist and treatment efficacy.

In their review of patient adherence literature, Goddard and colleagues (2020) highlighted motivation as one particularly influential individual factor on adherence to rehabilitation. This conclusion, combined with the consistent focus on perceived individual barriers (e.g., low selfefficacy, helplessness) in the physical therapy adherence literature, emphasizes the need to focus on patient motivation as a critical determinant of the physical therapy experience and rehabilitation process. 


\section{Patient Motivation and its Influence on Patient Adherence}

A number of studies (Chan et al., 2009; Chan et al., 2011; Chan \& Hagger, 2012) have examined the potential influence that motivation has on adherence in rehabilitation through the lens of Self-Determination Theory (Ryan \& Deci, 2000). Self-determination theory (SDT) is a motivation theory that addresses issues of personality development, self-regulation, universal psychological needs, life goals and aspirations, energy and vitality, nonconscious processes, the relations of culture to motivation, and the impact of social environments on motivation, affect, behavior, and wellbeing (Deci \& Ryan, 2008). Overall, it is concerned with the motivation behind choices that people make.

Cognitive evaluation theory (CET), as a sub-theory of SDT, suggests that intrinsic motivation (engaging in an activity for its own sake provided by the task itself), extrinsic motivation (engaging in an activity for instrumental reasons) and amotivation (absence of motivation) are three main categories of motivation within SDT. More specifically, CET states that that motivation can be divided into autonomous or self-determined motivation (e.g. interest and/or enjoyment in the activity) and controlled or non-self-determined motivation (e.g. guilt and/or external reinforcement) (Deci \& Ryan, 2000; Eynon, O’Donnell, \& Williams, 2017). Intrinsic motivation is reached when an individual participates in an activity because of reasons they identify. Extrinsic motivation is further divided into external regulation, introjected regulation, identified regulation, and integrated regulation to help us see how an individual's extrinsic motivation is regulated. External regulation satisfies an external demand or reward, introjected regulation behaviors are used to avoid guilt or anxiety along with enhance ego, identified regulation reflects personally valued or important activities, and integrated regulation confirm one's identity (Deci \& Ryan, 2008). 
As another sub-theory of SDT, the Basic Needs Theory suggests that autonomy, competence and relatedness are critical to facilitate self-determined motivation, social development, and personal well-being (Standage, Curran, \& Rouse, 2018). That is, satisfaction of individual autonomy (i.e., the need to experience activities as self-endorsed and purposefully enacted), competence (i.e., the need to interact effectively within the environment), and relatedness (i.e., the need to feel close, connected and cared for by others) can help individuals thrive and enhance physical and mental well-being, while thwarting or dissatisfaction of these needs can lead to individual frustration and a decrease in physical health and wellbeing (Standage et al., 2018).

Motivation has been identified to be an important consideration patient adherence to a physical therapy program. For PTs, once a patient feels that these needs are met, a shift to more self-determined form of motivation is likely to occur. Research done in identifying selfdetermined motivation is based off exercise adherence and what individuals need to do to reach a form of self-determined motivation. In the study by Silva, Markland, Minderico, Vieira, Castro, Coutinho, Santos, Matos, Sardinha \& Teixeira (2008), women were recruited from the community to meet in weekly or bi-weekly exercise intervention courses covering topics that are most successful for weight control. An autonomy-supportive climate was created for the participants to help them make autonomous decisions on exercise. Individuals with selfdetermined motivation were more autonomous in their exercise choices and more willing to continue with the exercise programs, leading to increased adherence to exercise. In physical therapy there is not much of an opportunity for patients to choose exercises in a rehabilitation program. Since this is the case, autonomy supportive behaviors can be expressed by patients through goals and exercises that they would like to do post-physical therapy. Ideally, patients 
would be able to flawlessly go through this shift in motivation, but most patients will need assistance. In order to help patients in reaching their ideal sources of motivation, PTs need to understand how these types of motivation can affect their patients in adhering to their rehabilitation program. Making this distinction can aide in facilitating motivation that creates autonomy in a patient and helps them adhere to their plan of care. In physical therapy there is not much of an opportunity for patients to choose exercises in a rehabilitation program. Since this is the case, autonomy supportive behaviors can be expressed by patients through goals and exercises that they would like to do post-physical therapy. To understand how PTs perceive and promote patient motivation, studies have focused on the perspectives of PTs themselves. Peek, Carey, Sanson-Fisher, \& Mackenzie (2017) examined perceptions that physiotherapists had about patient adherence. They found some patient characteristics that PTs identified as positive factors to patient adherence included self-motivation, self-confidence, belief the exercises will help and willingness to exercise.

A series of studies have focused on patient or athlete behavior with regard to rehabilitation. Chan and Hagger (2012), combined Self-Determination Theory and Theory of Planned Behavior model in their research and reported that autonomous motivation was positively associated with intention as mediated by attitude, subjective norms and perceived behavioral control. Furthermore, Chan et al., (2011) examined the SDT-based, trans-contextual model (TCM) to examine patient motivation. The TCM uses social cognitive and motivational theory that explains the transfer of one motivation from one context to another and is based on the SDT in which individuals have reasons or motives for their actions which are also called behavioral regulations (Chan, et al., 2011). The TCM suggests that perceived autonomy support from an agent (e.g., a PT) exerting an influence on an individual's autonomous motivation 
creates positive treatment motivation (Chan et al., 2011). The study by Chan et al., (2011) also found the same was true of non-self-determined motivation; if patient were not given the opportunity to be autonomous in treatment from their supporting agent, they were more likely to have negative treatment motivation based on the support they received. Patients that had increased autonomous motivation were more likely to adhere to their treatment and continue with physical therapy than those with more controlled motivation. The idea here is that the TCM gives evidence that autonomous sport motivation would form a positive association with positive treatment motivation with autonomy support from the physical therapist.

Finally, motivation in sports-injured patients have been found to be positively associated with attendance at sessions, completion on prescribed treatment, self-rated adherence, and selfreported completion of home exercise programs (Chan et al., 2009). Chan et al., (2009) investigated the impact of physiotherapists' autonomy-supportive behaviors on patient motivation and rehabilitation adherence after ACL surgery. The study found an indirect relationship with autonomy supportive behaviors on adherence and it accounted for $82 \%$ of the total effect. The study also reported that autonomous-support behaviors positively predicted treatment motivation and adherence was positively predicted by autonomous treatment motivation. On the other hand, treatment motivation was negatively predicted by controlled motivation. These factors of adherence are affected by the source of motivation the patient uses throughout therapy.

\section{The Importance of the Therapist and Patient Relationship}

To facilitate patient motivation, studies have identified the importance of the patient-PT relationship along with a range of strategies used to facilitate motivation (Kava, Fishleder, Steinman, Petrescu-Prahova \& Harris, 2019). Kava and colleagues (2019) investigated the 
barriers and facilitators to PA in older populations. The study was a randomized control trial using interviews with PTs and observations done in outpatient physical therapy settings. The observational data identified 4 categories that were important in interaction and communication between PT and patient; 1) building rapport, 2) PA type and frequency, 3) advice seeking and giving, and 4) resource sharing (Kava et al., 2019). As the most common barriers to treatment adherence have found to be low social support, low self-efficacy, pain, lack of knowledge, and time (Arnetz et al., 2004; Kava et al., 2019; O’Keeffe et al., 2016), building rapport between PT and patient is important to facilitate encouragement and positive reinforcement given to the patient through treatment.

Furthermore, facilitating patient involvement through skills such as goal setting can encourage a patient to adhere to their treatment plan. Arnetz and colleagues (2004) conducted a study designed to assess the effects of patient goal checklists, therapist goal checklists, and a goal forum checklist on patient involvement and motivation. These checklists gave patients and PTs a chance to create therapy goals for the patient and then compare and choose the best ones for the patient. Patients were also asked to fill out a questionnaire on the quality of care they received. The study suggested that patients who had a hand in creating treatment goals had better treatment outcomes compared to the patients in the control group. The intervention group was able to help set goals to aide or maintain through therapy if needed which was shown to have positive effects in client's compliance to treatment (Arnetz et al., 2004; Kava et al., 2019). Patient involvement in treatment increased patient motivation in physical therapy and home exercise programs. This can also be applied to athletes that are overcoming injuries as well. The most beneficial techniques for athletes were goal-setting and social support to create the basis for successful rehabilitation (Arvinen-Barrow et al., 2014). Thus, allowing the patient to be involved 
with some decisions in the treatment process can help them feel as if they are a part of the recovery, feel more comfortable in voicing their needs and give them a sense of autonomy in their treatment. (Arnetz et al., 2004; O'Keeffe et al., 2016).

Ultimately, the PT-patient relationship can help relieve some of these barriers to help a patient gain confidence in their ability to continue with therapy. Additionally, PTs can show individuals how to utilize the correct skills for their needs can benefit their progress and help them stick to their treatment plan. PTs can create rapport between patient and clinician so that patients can see the team effort that goes into reaching those goals rather than most of the burden falling on the shoulders of the patient or PT. Helping a patient identify the things that are holding them back can alter their mindset for what to expect from therapy. Furthermore, giving patients tools to increase their confidence and motivation in addition to therapists being receptive, present, and open can aide the patients as well (Miciak, Mayan, Brown, Joyce, \& Gross, 2018).

\section{Purpose of the Study}

Patient self-determined motivation, fueled by promoting patient autonomy, competence and relatedness, has therefore, been identified as an important positive predictor of treatment adherence. To help PT's further understand how to promote self-determined motivation in their patients, a closer examination of the specific factors and strategies influencing patient adherence and motivation is needed. Specifically, the purposes of this study were to explore PT's perceptions of: (1) the factors affecting patient adherence and motivation; and (2) the strategies and methods to facilitate patient adherence and motivation. This study sought to examine patient adherence and patient motivation through the lens of SDT and the BNT, with the guiding premise that if the basic needs are met and mentioned by PTs, then they are believed to aid intrinsic motivation and translate to patient adherence. 


\section{CHAPTER II: METHODS}

\section{Research Approach}

For this study, critical realism was used as the guiding philosophical framework, which diverges and lends from both positivist and constructivist paradigms (Denzin \& Lincoln, 2011; Fletcher, 2017). Critical realism posits that individual knowledge captures a small part of a deeper reality and treats the world as theory-laden but not theory-determined (Fletcher, 2017). Specifically, this approach suggests that theories help us get closer to reality and help us identify causal mechanisms driving social events or phenomena (Archer et al.,1998). As a research approach, critical realism was deemed appropriate because it can help researchers use a combination of theory and individual experiences to explain social events and suggest practical recommendations based to address social problems (Fletcher, 2017). Specifically, the focus of this critical realist research was examine the Self-Determination Theory (Deci \& Ryan, 2000) and the experiences and perceptions of physical therapists to understand patient adherence and motivation and the strategies use to motivated patients.

\section{Sampling Approach and Participants}

A purposive sampling approach (Robinson, 2014) was used to identify PTs that were contacted for interviews. PTs were selected based on the following criteria of: 1) being a licensed PT in the state of Illinois; 2) being a PT currently working in an outpatient physical therapy setting; and 3) having been working as a PT for at least one year in an outpatient clinic setting. Individuals who met the sampling criteria consisted of PTs in outpatient settings located in Illinois, which the primary researcher was familiar with. Following each participant interview, PTs were then asked at the end of their interview to pass along the interviewers' information to 
other PTs who could be interested in the study using snowball sampling as an attempt to increase sample size.

Thirteen physical therapists from outpatient physical therapy clinics throughout Illinois participated in interviews for the study. The PTs were all credentialed physical therapists from three different outpatient physical therapy clinics. Of the thirteen PTs, eight were female and five were male with a mean age of $31.3 \pm 3.1$ years of age. The PTs had an average of $5.7 \pm 3.5$ years of experience among them.

\section{Procedures}

Physical therapists considered for this research were based on the sampling criteria previously mentioned. Once participants were identified, they received an informational email regarding what the research entailed along with a consent form that was filled out prior to the interview. When consent of participation was received, the PT was contacted to set up a day, time and place for the interview to be conducted. Participants that participated were interviewed using semi-structured interviews with open-ended questions focused on PTs perceptions of patient adherence (Smith \& Sparkes, 2014). The form of interviewing done was individual semistructured interviewing with a preplanned interview guide focusing on a couple main ideas regarding adherence. Interviews were audio-recorded that allowed the researcher to go back and transcribe at a later date. The questions were designed to help PTs talk about experiences they recognized as adherence in their patients. Starting the interview with a conversational edge allowed the participant to establish a relationship with the interviewer (Smith \& Sparkes, 2014). The interviews were conducted using an interview guide created by the researcher and informed by previous research on patient adherence and motivation, as well as the SDT theoretical framework (Deci \& Ryan, 2000). 


\section{Semi-Structured Interviews}

The interview guide was designed with six main sections and is included in Appendix A. First, interviews started with questions focusing on participant's PT background and experience. These questions explored reasons why PTs wanted to become physical therapists, and followed with questions about their experiences as a PT, for example, "from your experience, what are some of your biggest challenges as a PT?" By commenting on their previous experiences, PTs were asked to explain what they found challenging in their profession and with their patients. Based on the answer that was given from challenges experienced, helped guide the next question that was asked by the interviewer.

The second section focused on perspectives of patient adherence. Questions about patient adherence explored how PTs perceived adherent versus nonadherent patients. For example, participants were asked, "Can you describe, from your experience, what an adherent patient looks like? What influenced this patient to be adherent? What common reasons do you hear for continuing with physical therapy?" when prompted about their experiences with adherent patients. They were also asked how they perceived nonadherent patients using the same type of question.

Third, the interview guide explored patient motivation. By connecting to earlier responses from participants, the interview questions asked participants to expand on their experiences and perceptions of patient motivation with the following question, "Please describe

the importance of patient motivation for patient rehabilitation." followed by "To what degree do individuals need to adhere to their rehabilitation protocols to achieve optimal rehabilitation outcomes?" This prompt/question allowed PTs to expand on their experiences with patient motivation and how that had shaped their current perceptions of the topic. 
Fourth, questions related to the relationship between a patient and their PT were focused on. The following question, "Does the PT-patient relationship affect patient adherence and motivation?" was followed by questions pertaining to why it is important and how to maintain that relationship with their patients.

Fifth, the interview guide progressed to questions related to strategies and methods that PTs have used to help their patients adhere and increase motivation to their physical therapy programs. In each interview, the interviewer related these questions to previous examples and experiences presented by the participants and followed-up with questions focused on whether PTs found strategies they had used successful or not and providing examples of successful and unsuccessful strategies used.

Sixth, and finally, participants were provided definitions of the three basic psychological needs (i.e., autonomy, competence and relatedness; Deci \& Ryan, 2000) and explicitly asked about their perceptions of how the three basic needs related to the outpatient physical therapy setting. Participants were asked how played a role in their perceptions of patient motivation and adherence and how their strategies to motivate patients aligned with the three basic needs. Explicitly asking PTs about the importance of the three basic needs allowed them to make connections back to previous questions asked and comment on which of the basic needs were important in facilitating patient adherence and motivation.

\section{Data Analysis}

A theoretical thematic analysis was used to gain rich social and psychological interpretations of the interviews, framed with an analytic interest in patient motivation (Braun \& Clarke, 2006). To find these themes, the theoretical thematic analysis used a deductively-driven approach to search across a data set to find repeated patterns of meanings (Clark \& Braun, 2006), 
that included the following phases: familiarizing yourself with the data, generalizing initial codes, searching for themes, reviewing themes, defining and naming themes, producing the report. First, the goal was to get familiarized with the data. Once all interviews were completed, they were reviewed and transcribed verbatim to create a script of each interview. The transcripts were accompanied by fieldnotes and memos that were taken during the interview to help in identifying themes portrayed in each. All transcripts and fieldnotes were read and re-read to gain an initial understanding of participant perspectives and experiences.

Second, the researcher sought to generalize initial codes by identifying raw units of data that addressed the two research questions by taking each interview transcripts and highlighting all information that pertaining to the purposes of the study. To address the first purpose of the study (i.e., PT's perceptions of the factors affecting patient adherence and motivation), initial codes were deductively identified to focus on individual/person-based and situational/environmental factors influencing patient adherence and motivation (Goddard et al., 2020). To address the second purpose of the study (i.e., PT's perceptions of the strategies and methods to facilitate patient adherence and motivation), initial codes were deductively identified that related to the three basic psychological needs (i.e,, autonomy, competence, and relatedness; Deci \& Ryan, 2000). This process culminated in theory-driven themes with lists of initial codes that represented all factors and strategies related to patient adherence and motivation.

Third, the search for themes started with the lists of initial codes organized in the theorydriven main themes (i.e., individual factors influencing motivation, environmental factors influencing motivation, strategies promoting autonomy, strategies promoting competence, strategies promoting relatedness). Next, each list of initial codes was reviewed to inductively identify sub-themes that provided further detail to answer the research questions. Data that didn't 
fit within these themes were placed in a miscellaneous sub-theme. Once the data was separated into sub-themes, it was reviewed for any other patterns that may arise from the interviews. This led to restructuring themes that were initially set and altering subthemes as needed.

Fourth, the main themes and sub-themes were reviewed, through the process of abduction. This involved re-described data using theoretical concepts and confirming that each theme and sub-theme accurately portrayed the participant perspectives and accurately aligned with the motivational theoretical tenets and SDT framework. The process of abduction assumes that a particular phenomenon or event that is interpreted from a set of general ideas or concepts yet accepts that theory is fallible and its level of description can be raised from individual perspectives (Danermark et al., 2002, p. 205; Fletcher, 2017).

Finally, all themes and sub-themes were defined and named to best describe the motivation factors and facilitation strategies. This phase was completed by charts created for each purpose to identify quotes that were similar, and themes were created on similar trends found in the data. Identifying the data that brought aspects of theory into realistic practice was the use of reintroduction. Reintroduction was used to identify the conditions for particular mechanisms to take effect because of the certain trends that are observed (Bhaskar, 1979, p. 32). This process helped move the reasoning process from concrete to abstract and back again to portray the approach that theories are used by in realistic approach but not driven by them (Lawson, 1998, p. 156). 


\section{CHAPTER III: RESULTS}

The purposes of this study were to explore PT's perceptions of: (1) the factors affecting patient adherence and motivation; and (2) the strategies and methods to facilitate patient adherence and motivation. The theoretical thematic analysis revealed that physical therapists had commonalities in what they perceived as their experiences with patient motivation and strategies they use to facilitate adherence in patients. PTs believed that patient's motivations were influenced by a range of individual and environmental factors. Specifically, four individual factors and four environmental factors were identified to influence patient motivation. The strategies and methods that PTs utilize in facilitating motivation and adherence were divided into the main themes of autonomy, competence, and relatedness with two sub-themes for each theme. Additionally, the importance of the physical therapy motivational climate was identified.

\section{Physical Therapists Perceptions of Factors Influencing Patient Motivation}

The first purpose of this study was to identify physical therapists' perceptions of factors influencing patient motivation. Based on the existing theoretical perspectives of participant and patient motivation (e.g., Deci \& Ryan, 2000; Goddard et al., 2020), factors influencing motivation can be categorized as both individual and environmental. When asked 'what factors usually influence whether a patient adheres to physical therapy,' individual motivational factors was more prioritized and discussed more frequently by physical therapists. The PT's placed greater emphasis on the patient controlling, managing and changing their own motivation, rather than factors in the PT and rehabilitation environment. Specifically, individual factors were focused on the responses from eight of the thirteen PTs $(61.5 \%)$ while five of the thirteen $(38.5 \%)$ focused on environmental factors and their influence on patient motivation. The two theoretically guided themes were then further divided into inductively identified sub-themes, 
with four individual factors and four environmental factors identified. Each sub-theme is presented below with supporting quotes for motivation factors that were mentioned.

\section{Individual Factors Influencing Patient Adherence and Motivation}

The individual theme was based on physical therapists' perceptions that there are preconceived ideas patients have coming into physical therapy and can affect their motivation throughout their rehabilitation process. The following four categories are individual factors that can affect patient motivation within physical therapy:

Patient Expectations. The participants believed patients that attend physical therapy have previous experiences or have heard what physical therapy might be like. Subsequently, these expectations affect how motivated they are to go to and continue with physical therapy. Expectations ranged from what patients thought would be happening during the session as told to them by their doctor or friends and family that have previously been to physical therapy. The following quote, for example, shows how PT's believe that some patients expect PT to be a passive kind of treatment where treatments would be done to them rather than them needing to be active in the treatment, supports that idea of a patient's expectation: "I think sometime people have the expectation that oh you go; you lay there and they do some stuff to you and you walk out and you're better in like 30 minutes." (DS).

While these patients may have a certain expectation about PT because of their initial meetings and beliefs, PTs believed it is important to help patients understand what the physical therapy process will look like to help alter that belief that the PT will solely run the treatment

process. When patients have the expectation that they have an active in the rehabilitation process, PT's believed that patients have greater adherence and motivation. This quote portrays that idea in the role that patient understanding and self-expectations have in physical therapy: 
"Especially in the sense of like getting people to understand that like PT is like a physical thing so they have to be the ones to do it so it's not gonna be you laying on the table doing a couple of joint mobilizations, like that's not the whole thing of it." (NC)

Explaining the process to a patient is important and was thought to help patients understand what their role in the process is as well.

Patient Valence. PT's stated that the reasons that patients come to physical therapy vary depending on whether they are post-surgical or not, have work-related injuries, or anything else in between they might be coming in for. For this reason, participants believed that patients valence drives their motivation to attend and progress through physical therapy. Specifically, patient valence was identified as an affective quality referring to the intrinsic positivity or negativity to the physical therapy experience and environment. PTs believed that for patients to be active in their treatment and do well with the process, they should have a positive mindset about physical therapy. This was expressed by the following PT when it came to patients coming to physical therapy:

There's some people that are not ready for physical therapy and don't really want to get better and then you know but besides that generally speaking most people do come here at the right time and they do want to get better. (EH)

Patients having that positive mindset coming into physical therapy can help patients feel more comfortable with the process. PT's noted that not all patients coming into physical therapy have that positive mindset, some may have a slightly negative view to the process due to previous experiences they have had. In the following example, this PT explains a patient's reasons for coming into physical therapy and why it's important to understand the patient's mindset and goals from the beginning: 
It wasn't like to decrease pain, it wasn't like to improve range of motion, it was like how does she get back to keeping her health as high as it can be, so that got her to buy-in. She was the one who was like you know I don't, she basically said she doesn't trust PTs. She had six visits and she didn't get better, okay six visits is nothing, but she didn't buy-in. $(\mathrm{RC})$

Notably, the patient was educated about her health and can express why she wants to be in physical therapy but is struggled with understanding the process and staying positive because of previous experience of not achieving progress and rehabilitation in a previous physical therapy experience.

Financial Resources. Resources patients have or have access to may affect their motivation to be successful in adhering and following through with physical therapy. These resources included insurance a patient has, their financial stability, ability to make appointments and more. One of the main factors PTs believed pertained to patient motivation was financial reasons. Many patients only have the ability to come to a handful of sessions because of their insurance premiums and can only afford so much physical therapy. An example of this was mentioned by LB where they mentioned several patients struggle with insurance and possible financial situation they are in:

Like, I had a 17-year-old kid that has a full-ride to [a Division I university], he wants to be a physician. He wants to play rugby, he tore his ACL playing football and he's having, had surgery, and before surgery I was like okay you're having surgery on Saturday; I'm giving you these exercises to do the day you get home, you need to start doing these now. And he was like oh wow, okay so do you think I can work on Sunday? Like he's always asking me when he can go back to work, he's a cashier at Walmart. And I was talking to 
$\mathrm{RC}$, because RC and I not necessarily share him but he's like treated him a couple times and he's like it really makes me wonder what the financial situation is in his house that he is that concerned with working a 12-hour shift at Walmart. (LB)

This PT explained that while they wonder about his financial situation at home, they can't tell him that it's okay to work since that goes against their ability to help him get better. This individual factor therefore posed a challenge to PTs and ability to treat their patients in the most beneficial way. PTs believed that in order to create the best plan of care for their patients they need to consider what is motivating them to be in therapy. LB also gave an example of a patient they have that is in the opposite financial situation, being very wealthy:

Flip the coin the other way, I have a patient who is very wealthy who had surgery and she came into surgery being pushed in a wheelchair in a cam-boot when she's weight bearing as tolerated. Like she could be up and walking and you know I asked what you've been doing for exercises and I mean she's young, healthy, very fit and she's like well I've been in the wheelchair just because it's easier and I have somebody to help me. I mean she has a chef, she has a butler like she can do all the things that she needs to do and it's not like she's like really my goal is to be able to walk again and I was like okay, step one: get your ass out of that wheelchair. Like you don't need to be in that wheelchair anymore like you can walk. (LB)

While this patient had the help and financial stability to take as much time as she wanted or needed to get better, the PTs found this to be a challenge as well. Participants perceived that patients who have resources available to them still need to be motivated to get back to their previous level of function. This also poses as a challenge to PTs since they want to find the best 
way to show patients what they can and can't do with where they are at in physical therapy right now.

Patient Education. The education a patient holds, from a PTs point of view, prior to coming into physical therapy is usually dependent on what they've heard from family, friends or what their doctor might tell them about the process. A patient's knowledge of their health and what physical therapy is can affect their motivation to continue with the process. PTs believed patients previous knowledge of physical therapy is important in whether or not they follow through with a physical therapy referral they are given. The following quote supports the idea that education may come by word of mouth from others rather:

I think education is a huge role because people might have heard from their doctor yeah I want you to go to physical therapy but yeah they might just have a general idea of what physical therapy is, they've never really done it before and don't really have family members or friends that have experienced it. (JT)

Multiple PTs expressed the importance of understanding their patient's knowledge of physical therapy prior to coming in. This knowledge was perceived by PTs as a starting point, where they would need to begin for their patients to understand the process. Participants agreed that education is essential in patient understanding of the process where both parties, patient and PT, are essential. PTs also expressed that based on patient knowledge of PT they expect a more passive kind of treatment that may lead to a quick fix of whatever is ailing them. Education was said to be important to discourage the idea that coming physical therapy is a quick fix to getting better:

So education is definitely a huge part but at the end of the day I think you know the instant gratification of our society to take a pill for that, do this you know like very 
quickly um is tough in our profession because we don't always you don't always get that kind of feeling.(ET)

Educating patients about how physical therapy varies from other health professions will help patients realize that physical therapy is a more active process than what is normally expected from other health care professionals. Understanding the level of knowledge patients have about physical therapy allows PTs to understand the motivation driving patients to be there. If a patient is there for a quick fix, their motivation may decrease once they realize they play a role in their progress and lead to a decrease in adherence. PTs perceived patient education as essential in helping patents understand their body and why the process takes times to help them return to their daily activities.

\section{Environmental Factors Influencing Patient Adherence and Motivation}

PTs believed that there are factors in the patient's environment, that they may not be able to control, that can affect their adherence and motivation throughout their rehabilitation process. This section presents the four sub-themes of environmental factors that can affect patient motivation within physical therapy:

Establishing a Relationship with the PT. The participants emphasized that the relationship between the PT and the patient can help the patient grow and become more confident in the process, in their PT, and their ability to go through with exercises given. PTs emphasized that they provide patients with a level of support through the process even if they may not from others in their family. These reasons showed participants believe that the rapport built between the PT and the patient is important in order to help a patient feel comfortable with the therapy process, that is supported with the following quote: "The reason I say that, I think 
like if you have a good relationship with somebody, they're going to trust you, they're going to believe you. Maybe they a little more buy-in and do the stuff you ask of them.” (DS)

PTs believed that this relationship helps garner and build patient trust in their PT and the therapy process. Patients could be more willing to buy-in and have a positive experience once that rapport and trust is built. The connection that is established shows patients their PT cares about their progress and that they want the patient to do well with the process, which is supported by this quote:

So, if you're able to establish like um a connection with somebody and show them that I care about you and what you're doing and they feel connected to you because of that they feel like it's almost their responsibility to make the therapist proud in a way. (TD)

The PT-patient relationship was believed by participants to establish a connection that elicits a positive response from the patient. That positive response is due to patients seeing that their PT cares about how they are doing and in return want to show their PT the progress they've made.

Clinic Environment. Participants stated that the clinic environment can play a role in how patients feel in the setting. A clinic can be big, loud, and busy while others can be smaller, quieter and more relaxed. Depending on the type of setting a patient steps into affects their ability to progress and complete physical therapy. Participants believed that the clinic environment that is present could be challenging for patients to navigate and keep from being successful throughout their program. A challenging environment was described by some PTs to not have accommodations some patients might need, such as a private treatment room if they don't feel comfortable in an open gym area. Depending on the patient and how they react to an environment could lead to them not being able to progress: "And if they come in and they're like 
oh snap what is going on here, then they're like I'm out I can't. It's not because they're not compliant, we didn't provide an environment for them to be compliant." (TD)

The PT and the patient are put in a challenging position because the environment of the clinic can't be changed. Participants discussed that some clinics have private treatment rooms that they use if needed but those that don't have those accommodations, may keep the patient from being able to succeed. Responsibility can then fall on the PT to create that comfortable environment for the patient so that they can be successful. Patients that feel comfortable in the clinic they are in are believed to enjoy and feel successful in the process:

I think that if they enjoy the environment, you enjoy your surroundings then it's just you're more likely to do it and I think if you trust us then you trust that we're saying do this twice a day five days a week, then that you're more likely to do it because we're people you trust. (NC)

Participants agreed that creating a comfortable and enjoyable environment for patients can help them enjoy being in a clinic and not feel like they are sterile environment like other doctors' offices.

Geographic Accessibility to PT Services. The community or environment a person lives in can play a role in their ability to adhere to physical therapy. Accessibility to healthcare, transportation, recreation centers, and neighborhood are some of the factors that can play a role in a patient's motivation to adhere to physical therapy. Multiple PTs voiced the importance of considering the access they have to be able to continue exercises at home: "A lot of times socioeconomic status is involved too, they don't have access to a gym or some place to be compliant." (KS) 
PTs described the importance of making sure they are aware of what their patients have access to so that they can give them home exercise programs with what they have can complete with resources available to them. Sometimes for PTs to realize this, their patients give it as a reason for why they aren't able to continue with home exercises:

Like I've had a handful of patients where I tell them, you need to go out and go for a walk for like 30 minutes a day. And that's what I'll ask them to do for home, but they're like it's not safe for me to go and walk around for 20 minutes a day. And they don't have the money to join a gym or they don't feel comfortable to like their local rec center if they even have one. (DS)

PTs believed that understanding the community a patient resides in and what they can access could completely change the way their PT educates them in what to do outside of the clinic. This was seen as educational for both the PT and their patient in order to find modifications that can help the patient still be successful with what they have available to them.

Family and Vocational Commitments. PTs recognized that patients' motivations to be in and continue with physical therapy was affected by their commitments to their families and work. Participants stated that some patients may not have time to include physical therapy into their daily routine because they have multiple jobs, they are taking care of their children, or they are caring for a sick family member. Participants expressed other important commitments to be a barrier for a patient to prioritize physical therapy:

Like if you have a lot of family stuff going on then it's really hard too, like I was talking to you about my patient who has had, every time she has come in she'd got a new person close to her or a relative or somebody that has died and another health issue and 
insurance stuff going on. I mean it's hard to help someone heal when they've got all these other barriers in the way and that's not necessarily their fault. (LB)

PTs described the importance of understanding that patients want to get better through physical therapy, but they feel like they don't have the ability to because of other things happening in their life. PTs expressed that this is something patients rarely have control over and that they need to remember that sometimes it's not the person that isn't adherent but the situation they are in is keeping them from being so. PTs understand that patient adherence can be heavily impacted by other commitments that a patient has.

It's not because they don't wanna do it, like maybe they have a different home situation, maybe they have four kids, work two jobs and they get home and the last thing they want to do is quad sets. Um so I think compliance is heavily impacted by someone's personal life. (TD)

\section{Physical Therapists Perceptions of the Strategies and Methods to Facilitate Adherence and Motivation}

The second purpose of this study was to identify physical therapists' perceptions of the strategies and methods used to facilitate motivation and adherence. PTs were asked what strategies they had previously used in facilitation, whether they were successful or not. When identifying strategies and methods PTs made both explicit and implicit mentions of the three basic psychological needs of autonomy (the need to feel ownership of one's behavior), competence (the need to produce desired outcomes and to experience mastery) and relatedness (the need to feel connected to others) before definitions of the needs were given (Deci \& Ryan, 2000). Every PT interviewed mentioned at least one of the basic needs before being given formal definitions. Specifically, 9/13 (69.2\%) PTs mentioned autonomy, 8/13 (61.5\%) mentioned 
competence and 9/13 (69.2\%) mentioned relatedness before knowing the definitions of them. From this we were able to identify the three basic needs as the three themes that encompassed the strategies and methods given along with subthemes arising from each basic need.

\section{Autonomy}

Autonomy is the need to feel ownership of one's behavior (Standage et al., 2018). Participants believed that, for patients to feel ownership over their behavior in physical therapy, it begins at the initial evaluation when the PT and patient meet. During this visit the patient and PT establish expectations for both parties and a plan of care that will help patients return to normal function. By establishing these expectations, patients know what they can do at every step of the process. With this in mind, two subthemes of autonomy were identified to directly explain the strategies used for facilitating motivation and adherence; promoting patient buy-in and educating to take control of the process.

Promoting Patient Buy-In. Participants emphasized that patient buy-in is essential for patients that want to succeed in physical therapy. Patient buy-in is promoted by physical therapists throughout the entire rehab process, but that initial evaluation is believed to be the most important session when it comes to telling patients what they can expect from physical therapy and connect with their physical therapist. Promoting patient buy-in allows physical therapists to educate patients in what to expect from the process and gives patients space to lay out their expectations as well. PTs believed that patient buy-in was expressed as an important part in facilitating autonomy in a patient. The following quote reflects this idea:

And then um like you were saying within the evaluation, setting them up from the very beginning to help them understand physical therapy; what is our goal, you know, what does physical therapy entail and I think it's important too to almost let people know like 
we might be doing some more hands-on techniques initially but our goal is to progress you until you're doing exercises on your own. (JT)

By setting patients up with expectations, participants stated that it allows patients to see what they need to be able to accomplish and what to look forward to from the process. PTs claimed that they continuously try to facilitate patient buy-in in when they are educating the patient, showing patients exercises, giving them updates on measurements or if they're reaching a certain point in their protocol. PTs believed the importance of patient buy-in is to help them see that the process works, and they will get better since the process has worked previously.

Educating to Take Control of the Process. PTs believed that they can help their patients become motivated by educating them about the physical therapy process. Patients coming to physical therapy may not know what to expect and their PT can help with that uncertainty through education and highlighting milestones throughout the process. Participants emphasized that educating patients about the process is critical to helping patients learn how to take control over their own health and feel confident in their ability. Participants believed that when patients are able to accomplish tasks they haven't done since their first physical therapy visit helps them realize that therapy is helping them do things they once did:

That's a huge deal for somebody and it's what most people would consider a simple thing, if you can't do it I mean you're losing your independence and they come in and they are like yeah I can almost put my shoe and sock on now. Trying to explain to them how large of a deal that is, that they're making progress in things like that. (DF)

Explaining to patients that even the smallest milestones can lead to bigger accomplishments through physical therapy was believed to be important. PTs believed that helping patients see even the smallest things, like being able to put on a sock and shoe, brings 
them a step closer to the independence patients are seeking, thus facilitating their motivation. Realizing these milestones could help patients see that improvement is in their hands and they need to take control of the process and enhance self-motivation. Focused on educating patients, this subtheme was also inherently linked to perceived competence.

\section{Competence}

Competence is the need to produce desired outcomes and to experience mastery (Standage et al., 2018). In physical therapy, participants described that competence can be enhanced when patients successfully completing exercises during their in-clinic session, completions of given home exercise programs and more. PTs believed that they can facilitate competence in their patients through patient education about why they are in physical therapy and why physical therapy could help. Competence is also facilitated by constant reminders of patient progress from their PT. Participants believed that when patients can see how they are progressing, whether it be an increase in range of motion or making an exercise more challenging, it helps them see that they can reach their desired outcomes through mastery of certain tasks. This was how two subthemes of competence, supporting patient progress and patient education, were identified.

Supporting Patient Progress. Participants emphasized that patient progress in physical therapy is constantly on the mind of both the PT and the patient. How PTs monitor patient progress is expressed to patients from their very first visit so patients are aware of expectations in therapy. While measurements and protocol are important in progress, participants noted that it is important for PTs to support their goals and activities that they would like to return to. PTs believed that supporting their patients progress through physical therapy allows patients to feel that they are doing what they need to be to get back to what they were previously doing: 
I think it's really helpful for me to understand like what they're doing outside the clinic because it helps me understand their goals better...like if you want to be able to sit at a grandson's football game, I know that's a goal for you and I feel like I understand you a bit better. (NC)

This PT explained the importance of understanding a patient's goals and catering their treatment to their goals. Catering to the patient's ability and progress while keeping in mind what stage in protocol they might be enables patients to feel they are being heard in the process. PTs supporting their patients this way can help them see that their progress is related to a goal that matters to them rather than something they were told to do. When a patient can see that they have the support of their PT throughout physical therapy, PTs expressed they saw increased motivation in their patients to do well and better adhere to their program.

Patient Education. PTs recognized that patient education is critical in helping understand why they are in physical therapy to begin with. By assuming patients do not know a lot about physical therapy or their injury, the PT can establish what is important for the patient to know and what they need to do to be successful, and subsequently enhance patient competence. Starting with basics (e.g., explanation about a surgery that was done, muscles involved in the injury and exercises done, protocol used for certain injury or surgery, etc.) allows the PT to fully explain methods they use and why the process works the way it does. Educating patients about their injury or whatever they are in physical therapy for allows them to understand how the process, and their participation, can help them get back to their normal:

Um in the beginning you think like you want to do everything and you wanna you know solve the patients problems and things like that and then I think I realized more over time 
that it's super important to teach them how to rehab themselves and to give them the confidence and the education ability to be able to do that on their own. (LD)

PTs expressed that wanting to solve the patient's problem can lead to other problems along the way. It is important to teach patients everything that is happening to them in laymen terms in order for them to understand and be able to follow the process. When PTs can successfully educate their patients about their body and injury, patients can see how the PTs plan their treatment based off patient needs. Teaching patients why certain exercises are chosen for them can help patients understand that they are doing them for a reason, and they can become more competent in their ability to perform said exercises, thus enhancing adherence to the exercise program

\section{Relatedness}

Relatedness is the need to feel connected to others (Standage et al., 2018). In physical therapy, relatedness was described as the rapport built between patient and PT and building relationships amongst patients in physical therapy. The idea of having a form of social support is why PTs felt relatedness was important within physical therapy. PTs are unique in that they see patients' multiple times a week for weeks or months at a time. This is the reason they found relatedness to be important since they need to build rapport with their patients to gain their trust and belief in the process for a certain amount of time. In addition to that, patients could be around others that have similar injuries or disabilities where they will come to support one another. From this, the following sub-themes were created, building rapport and patient to patient relationships.

Building Rapport. From the initial evaluation, PTs establish who they are as a clinician and as a person when communicating with their patient. The participants believed that the 
relationship between the patient and their PT is significant since patients are being seen more often by their PT than any other healthcare professional. PTs believed that building rapport assists them in being able to understand a patient's motivations and goals for being in physical therapy to help them reach their full potential. Finding those commonalities between the patient and PT can help keep patients engaged in sessions and help ease their minds at the prospect of being in a clinic setting for so long:

I mean, I know it's funny that I talk about food in here all the time but that's one thing that everyone has in common in some way shape or form. Whether they have the same diet or not, again, it's something that can be discussed and so it's like using something like that to find a way to start to have a conversation, I feel, helps ease people's minds a little bit and forget about the anxiety of being in a doctor-like office setting. (DF).

PTs recognized that finding that one thing in common with a patient helps them feel comfortable within the environment and their PT. Establishing that relationship gives patients a chance to trust their PT and their ability to cater their program to their needs. This could lead to patients wanting to please their PT with progress they are making and showing them that they are part of this process as well instead of just relying on the PT.

Patient to Patient Relationships. PTs discussed how important it was to also consider patient to patient relationships within physical therapy. When someone attends physical therapy, there are other patients that work with the same PT or another PT within the clinic same clinic. Patients tend to talk to others around them and find that might have similar injuries to theirs and will create relationships between one another. Patients can encourage one another and establish relationships through similarities such as injury, background and even just appointment time. Participants believed that interactions between patients help them receive another type of support 
from someone who may know what they are going through: "I encourage my patients to interact with patients nearby. Especially with our older geriatric patients, a lot of them don't want to leave because they get to socialize back here. And that just helps healing I feel like.” (RC)

While PTs were split among how they felt about patient to patient relationships, most decided that these relationships can be important to some patients. In the quote, this PT mentioned that they feel it's important for patients to socialize since they feel it helps with healing. Having others that a patient can relate to is important since they will have someone, other than their PT, to talk about the physical therapy process. While this may appear discouraging depending on the patients' progress, it was believed to help to motivate patients to reach their goals.

\section{The Importance of the Motivational Climate}

In the final question of the interview, participants were asked to describe the importance of the three basic needs in order to facilitate adherence followed by their ranking of basic needs. In the final answers, autonomy was ranked first by 10 of 13 (76.9\%) PTs, competence was ranked first by 1 of 13 (7.7\%) PTs and relatedness was ranked first by 2 of 13 (15.5\%) PTs. The majority of the PTs cited autonomy as most relevant basic need in facilitating adherence and motivation because they felt that patients need to have a sense of ownership over their own progress and treatment to be successful. While these were the rankings given by the PTs, most said that the rankings could depend on which patient they are treating and what they might need from the PT during physical therapy. Collectively, these responses emphasized the importance of understanding both individual and situational factors influencing patient adherence and motivation. PTs discussed how satisfying the basic psychological needs was situational rather than the same for every patient coming into physical therapy. Additionally, while rankings were 
provided, it was especially notable that participants described the importance and satisfying all, and not just one, of the basic psychological needs. This highlighted their perception to help to establish a needs satisfying motivational climate to facilitate of adherence and motivation. Specifically, in order to facilitate self-determined motivation, PTs expressed that the using the basic needs in different combinations could be the most effective way to facilitate adherence and motivation. Participants examples of how to promote autonomy-competence, competencerelatedness, and relatedness-autonomy helped shape their views of an effective motivational climate.

For example, to promote both autonomy and competence, EH describing promoting selfcontrol and education together, "Mostly just helping them realize everything that they're capable of or I like to really put the patient in the forefront of their own care and helping them realize how independent they can be with this stuff." To promote competence and relatedness, LB discussed the importance of building rapport and providing positive encouragement when interacting with patients:

You get to know the people that you come with and like hey you look really good! She's got you working hard today! Like that kind of stuff and then being competent with your exercises, like if I have to explain to somebody how to do a sit to stand every time something is wrong. (LB)

Finally, to satisfy relatedness and autonomy together, DS discussed building a "buddy buddy" system to help patients feel in control:

You get people who come in at like similar times all the time or are like working towards similar goals, have the same procedure. Like people get pretty buddy buddy and are 
almost like motivating factors for one another and you almost see a little bit of a healthy competition between patients, which I think is really good. (DS) 


\section{CHAPTER IV: DISCUSSION}

The purpose of this study was to (1) explore PTs perceptions of the factors affecting patient motivation and (2) PTs perceptions of strategies and methods used to facilitate adherence and motivation in patients. The results expressed the different factors PTs believe their patients face when it comes to their motivation before and during physical therapy. PTs perceived factors affecting patient motivation to fall into individual and environmental factors. Individual factors included patient mindset, patient expectations, patient education while environmental factors included clinic environment, geography and family commitments. Additionally, this study identified a range of strategies and methods that PTs used to promote patient autonomy, competence and relatedness, to ultimately facilitate patient adherence and motivation

These themes and subthemes of patient motivation helped us understand what physical therapists perceived as important for patients in physical therapy. It was particularly interesting that findings indicated that PTs perceived individual factors influenced patient adherence and motivation to a greater degree than environmental factors. Through their literature review, Goddard and colleagues (2020) found that adherence is influenced by the two personal and situational components. Subsequently, using these categories in our deductive analysis, specific individual and environmental factors were identified that affect patient adherence and then motivation. While the PTs had perceptions of what motivating factors were for their patients, the collective findings highlighted that PTs believed that patient motivation is primarily patient dependent. This supports the presence of personal and situational components affecting patient motivation and adherence in rehabilitation settings (Goddard, 2020), yet provides a unique finding that individual patient factors may need be a scientific and practical focus. Even though the PTs can influence a patient's motivation, they believe patient motivation varies and depends 
greatly on what the patient is experiencing (Sluijs et al, 1993). It is important for PTs to recognize factors that affect their patient's motivation in order to better cater treatment to their needs.

In order to understand how PTs cater treatment to patients, they identified strategies and methods they used to facilitate patient adherence. PTs identified strategies and methods to include aspects of the basic needs before they were told the formal definition of each. PTs strategies focused on what the patients could improve on or what the PT could do to facilitate motivation and adherence. They were focused on supporting the patients and giving them the ability to be successful in physical therapy. The PTs identified strategies that are important to consider since other studies highlight the importance of individuals identifying the strategies used to foster positive adherence (Goddard et al., 2020). Having the PT perspective helps emphasize strategies that they have had success with and how they can improve on other strategies that might not have been successful. This portrays that rehabilitation is constantly changing and there isn't one certain strategy that works for each individual, even if they have the same ailment.

The strategies identified by the PTs were placed into the themes of autonomy, competence and relatedness. These themes allowed us to understand what PTs felt was important when trying to facilitate motivation and adherence in their patients. Strategies that were identified under the theme of autonomy, focused on patients being aware of and understanding the physical therapy progress. PTs identified including patients in programming, goals, and the process in general was seen to be a successful strategy for PTs when facilitating motivation and adherence (Peek et al., 2016). PTs expressed that using autonomy-supportive strategies in physical therapy created a more self-determined motivation in patients (Chan et al., 2011; Deci 
\& Ryan, 2008). Those that were identified under the theme of competence highlighted patient education and supporting their progress. PTs emphasized that patient education was important in understanding the process they are going through. In addition to understanding that process, it was also important to support patients progress through their rehabilitation. Strategies identified under the relatedness theme were identified as building rapport and building patient to patient relationships (Kava et al., 2019; Ryan \& Deci, 2000). Finding those little similarities between either PT or patient can help an individual create a relationship with someone that can help increase that motivation to progress through physical therapy. These themes help us identify a motivational climate which expressed interesting findings of the basic needs multifaceted uses in physical therapy. When participants were asked to rank the basic needs of autonomy, competence and relatedness, most would give a ranking and then mention that it would be dependent on what a patient would need. In the sense, identifying the needs as patient dependent and that a mix of them could be necessary to create an environment where they can facilitate motivation and adherence in their patients. These themes helped us identify strategies that enabled patients to foster self-determined motivation helped them achieve success in physical therapy (Chan et al., 2009). Finding a strategy that is successful is patient dependent so one strategy that works for a certain patient doesn't necessarily mean it will work for another. PTs are constantly changing their strategies and relying on what their patients need in order to give them the best care they can and facilitate motivation and adherence.

\section{Practical Recommendations}

Practical recommendations that could be considered for future professionals could be creating further continuing education courses for PTs pertaining to the use of basic needs in treatment and creating paperwork that can be used to evaluate barriers to patient motivation and 
adherence. Creating or altering current continuing education courses for PTs and other professionals in a rehab setting can allow for better understanding in how they can facilitate adherence and motivation utilizing autonomy, competence and relatedness (Zalewski et al., 2014). Based on the findings, it would be beneficial to create in clinic continuing education for PTs to utilize based on the motivational climate that was formed. These in clinic practices could be based on in-services, or presentations, done by the clinicians to learn more about how this climate could affect their patients. These presentations are already done for continuing education based on musculoskeletal research and the same could be done with the utilization of the basic needs. In these settings, clinicians could also be able to bring in patients, like a case study, to discuss how the use of these needs could benefit the patient based on current factors affecting them. They can discuss how patients may be affected by individual and environmental factors they're facing to help them overcome those barriers. This continuing education would allow PTs to learn how to utilize these needs correctly and better be able to recognize which need would be more beneficial than another to facilitate motivation and adherence.

Additionally, there are questionnaires and surveys PTs utilize that give patient reported measures of adherence while those that are based on clinician reported measures focus on patient participation during sessions (McLean et al., 2017). Questionnaires and surveys currently used focus on either the clinician or patients reports, not taking both reports into consideration. Creating a questionnaire or outcome survey that takes into consideration clinician and patient reported measures, can enhance a PT's understanding of the individual psychological factors influencing motivation and lead to better measures of adherence and motivation. This could also lead to better conversations between patient and PT for both parties to understand what might be keeping a patient from being adherent to physical therapy. 


\section{Limitations and Future Research}

The study followed a critical realist approach that allowed us to find connections between theory and practice. A strength of this approach was the ability to see the use of the theories applied in the study working in the physical therapy setting. Participants were able to express perceptions, strategies and methods relating to the theories that supported this study. This approach allowed us to make connections between the theories used and the data that was collected through interviews. A limitation of this approach was that while the idea of the theory approach was appealing to the PTs, they felt that it would be difficult to use that in practice. It seemed as though they felt that the theories helped explain the ideas we were discussing and could lead to infinite mechanisms that drive the outcomes of practice.

While a qualitative approach to this study provided detailed examples about the factors influencing patient motivation and the strategies to enhance motivation, the purposive sampling and findings only present the unique experiences of a small sample of PTs and cannot be generalized to all therapists. The use of snowball sampling was unsuccessful since the researcher did not receive additional participants outside those recruited using purposive sampling. Further research could use survey-based research across a larger number of PTs to examine their approaches to supporting patient's basic psychological needs and forms of motivation, and thus, provide generalizable insights. Future research could also be done from this study could including patient perceptions as well to compare what each party believes is important when it comes to motivation and adherence. Including both patient and PT perspectives could help us identify if there is anything else to consider important in the rehabilitation setting. 


\section{Conclusion}

In this study we were able to consider physical therapists' perceptions of patient motivation and adherence. The findings helped us gain an understanding of the factors that have been said to affect a patient's motivation and adherence to rehabilitation. Identifying strategies and methods used in facilitation of motivation and adherence is beneficial in future practice for physical therapists. It is important to consider how clinicians perceive their patient's actions and beliefs in order to create a positive environment for progress and rehabilitation. Further research needs to be done in order to fully understand the implications of these findings. 


\section{REFERENCES}

Archer, M., Bhaskar, R., Collier, A., Lawson, T., \& Norrie, A. (1998). General introduction. In M. Archer, R. Bhaskar, A. Collier, T. Lawson, \& A. Norrie (Eds.), Critical realism: Essential readings (pp. ix-xxiv). London; New York: Routledge.

Arnetz, J. E., Almin, I., Bergström, K., Franzén, Y., \& Nilsson, H. (2004). Active patient involvement in the establishment of physical therapy goals: Effects on treatment outcome and quality of care. Advances in Physiotherapy, 6(2), 50-69. https://doi.org/10.1080/14038190310017147

Arvinen-Barrow, M., Massey, W. V., \& Hemmings, B. (2014). Role of Sport Medicine Professionals in Addressing Psychosocial Aspects of Sport-Injury Rehabilitation: Professional Athletes' Views. Journal of Athletic Training, 49(6), 764-772. https://doi.org/10.4085/1062-6050-49.3.44

Babatunde, O. O., Jordan, J. L., Van der Windt, D. A., Hill, J. C., Foster, N. E., \& Protheroe, J. (2017). Effective treatment options for musculoskeletal pain in primary care: A systematic overview of current evidence. PLoS One, 12(6).

Bhaskar, R. (1979). The possibility of naturalism: A philosophical critique of the contemporary human sciences. Atlantic Highlands, N.J.: Humanities Press

Braun, V., \& Clarke, V. (2006). Using thematic analysis in psychology. Qualitative research in psychology, 3(2), 77-101.

Brewer, B., Cornelius, A., Van Raalte, J., Petitpas, A., Sklar, J., Pohlman, M., Krushell, R. J., \& Ditmar, T. D. (2003). Protection motivation theory and adherence to sport injury rehabilitation revisited. The Sport Psychologist, 17(1), 95-103. https://doi.org/10.1123/tsp.17.1.95 
Chan, D. K., Lonsdale, C., Ho, P. Y., Yung, P. S., \& Chan, K. M. (2009). Patient Motivation and Adherence to Postsurgery Rehabilitation Exercise Recommendations: The Influence of Physiotherapists’ Autonomy-Supportive Behaviors. Archives of Physical Medicine and Rehabilitation, 90(12), 1977-1982. https://doi.org/10.1016/j.apmr.2009.05.024

Chan, D. K.-C., Hagger, M. S., \& Spray, C. M. (2011). Treatment motivation for rehabilitation after a sport injury: Application of the trans-contextual model. Psychology of Sport and Exercise, 12(2), 83-92. https://doi.org/10.1016/j.psychsport.2010.08.005

Chan, D. K., \& Hagger, M. S. (2012). Self-determined forms of motivation predict sport injury prevention and rehabilitation intentions. Journal of Science and Medicine in Sport, 15(5), 398-406.

Danermark, B., Ekström, M., Jakobsen, L., \& Karlsson, J. C. (2002). Explaining society: An introduction to critical realism in the social sciences. London: Routledge.

Deci, E. L., \& Ryan, R. M. (2008). Self-determination theory: A macrotheory of human motivation, development, and health. Canadian Psychology/Psychologie Canadienne, 49(3), 182-185. https://doi.org/10.1037/a0012801

Denzin, N. K., \& Lincoln, Y. S. (2011). The Sage handbook of qualitative research. Thousand Oaks: Sage.

Eynon, M. J., O’Donnell, C., \& Williams, L. (2017). Assessing the impact of autonomous motivation and psychological need satisfaction in explaining adherence to an exercise referral scheme. Psychology, Health \& Medicine, 22(9), 1056-1062. https://doi.org/10.1080/13548506.2016.1274041

Fletcher, A. J. (2017). Applying critical realism in qualitative research: methodology meets method. International Journal of Social Research Methodology, 20(2), 181-194. 
Goddard, K., Roberts, C., Byron-Daniel, J., \& Woodford, L. (in press). Psychological factors involved in adherence to sport injury rehabilitation: a systematic review. International Review of Sport and Exercise Psychology,

123. https://doi.org/10.1080/1750984x.2020.1744179

Jack, K., McLean, S. M., Moffett, J. K., \& Gardiner, E. (2010). Barriers to treatment adherence in physiotherapy outpatient clinics: A systematic review. Manual Therapy, 15(3), 220228. https://doi.org/10.1016/j.math.2009.12.004

Kava, C. M., Fishleder, S., Steinman, L., Petrescu-Prahova, M., Schrodt, L., \& Harris, J. R. (2019). Provider-Patient Communication and Involvement in Physical Activity Programs Among Patients Receiving Physical Therapy Services: A Qualitative Study. Journal of Applied Gerontology, 073346481984740. https://doi.org/10.1177/0733464819847402

Lawson, T. (1998). Economic science without experimentation. In M. Archer, R. Bhaskar, A. Collier, T. Lawson, \& A. Norrie (Eds.), Critical realism: Essential readings (pp. 144169). London; New York: Routledge.

McLean, S., Holden, M. A., Potia, T., Gee, M., Mallett, R., Bhanbhro, S., ... Haywood, K. (2017). Quality and acceptability of measures of exercise adherence in musculoskeletal settings: A systematic review. Rheumatology (Oxford, England), 56(3), 426-438. https://doi.org/10.1093/rheumatology/kew422

McLean, S. M., Burton, M., Bradley, L., \& Littlewood, C. (2010). Interventions for enhancing adherence with physiotherapy: A systematic review. Manual Therapy, 15(6), 514-521. https://doi.org/10.1016/j.math.2010.05.012 
Miciak, M., Mayan, M., Brown, C., Joyce, A. S., \& Gross, D. P. (2018). The necessary conditions of engagement for the therapeutic relationship in physiotherapy: An interpretive description study. Archives of Physiotherapy, 8(1). https://doi.org/10.1186/s40945-018-0044-1

O’Keeffe, M., Cullinane, P., Hurley, J., Leahy, I., Bunzli, S., O’Sullivan, P. B., \& O’Sullivan, K. (2016). What Influences Patient-Therapist Interactions in Musculoskeletal Physical Therapy? Qualitative Systematic Review and Meta-Synthesis. Physical Therapy, 96(5), 609-622. https://doi.org/10.2522/ptj.20150240

Peek, K., Carey, M., Sanson-Fisher, R., \& Mackenzie, L. (2017). Physiotherapists’ perceptions of patient adherence to prescribed self-management strategies: A cross-sectional survey of Australian physiotherapists. Disability and Rehabilitation, 39(19), 1932-1938. https://doi.org/10.1080/09638288.2016.1212281

Robinson, O. C. (2014). Sampling in interview-based qualitative research: A theoretical and practical guide. Qualitative research in psychology, 11(1), 25-41.

Ryan, R. M., \& Deci, E. L. (2000). Self-determination theory and the facilitation of intrinsic motivation, social development, and well-being. American Psychologist, 55(1), 68-78. https://doi.org/10.1037/0003-066X.55.1.68

Silva, M.N., Markland, D., Minderico, C.S. et al. A randomized controlled trial to evaluate selfdetermination theory for exercise adherence and weight control: rationale and intervention description. BMC Public Health 8, 234 (2008). https://doi.org/10.1186/1471$2458-8-234$

Sluijs, E. M., Kok, G. J., \& Van der Zee, J. (1993). Correlates of exercise compliance in physical therapy. Physical therapy, 73(11), 771-782. 
Smith, B., \& Sparkes, A. C. (Eds.). (2014). Qualitative research methods in sport, exercise and health: From process to product. Routledge.

Standage, M., Curran, T., \& Rouse, P. C. (2018). Self-Determination-Based Theories of Sport, Exercise, and Physical Activity Motivation. In Advances in Sport Psychology (4th ed.). Human Kinetics.

Tuakli-Wosornu, Y. A., Selzer, F., Losina, E., \& Katz, J. N. (2016). Predictors of Exercise Adherence in Patients With Meniscal Tear and Osteoarthritis. Archives of Physical Medicine and Rehabilitation, 97(11), 1945-1952.

https://doi.org/10.1016/j.apmr.2016.05.011

World Health Organization. (2003). Adherence to long-term therapies: evidence for action. World Confederation of Physical Therapy. (2016). What is Physical Therapy?

Zalewski, K., Alt, C., \& Arvinen-Barrow, M. (2014). Identifying barriers to remaining physically active after rehabilitation: Differences in perception between physical therapists and older adult patients. journal of orthopaedic \& sports physical therapy, 44(6), 415-424. 


\section{APPENDIX A: INTERVIEW GUIDE}

1. Introduction of study and participants

a. Please describe your PT experience

i. How many years as a PT and how many years with current facility?

b. Why did you become a PT?

2. From your experience, what are some of your biggest challenges as a PT?

3. Please describe the importance of patient motivation for patient rehabilitation.

a. To what degree do individuals need to adhere to their rehabilitation protocols to achieve optimal rehabilitation outcomes?

4. Can you describe, from your experience, what an adherent patient looks like?

a. What influenced this patient to be adherent?

b. What common reasons do you hear for continuing with physical therapy?

5. Can you then describe what a non-adherent patient looks like?

a. What kept them from being adherent?

b. What common reasons do you hear for not continuing with physical therapy?

6. From your experiences, what factors usually influence whether a patient adheres to physical therapy or not?

a. What barriers do patients face in becoming adherent?

7. Does the PT-patient relationship affect patient adherence and motivation?

a. If so, what about the relationship is important for patient adherence?

b. If so, what do you do as a PT to develop and maintain the relationship?

c. If not, why not?

8. What specific strategies do you use to help patients adhere to their physical therapy program?

a. Have these strategies proved successful in the past?

i. Please provide examples explaining when and why they were successful

ii. If they were not successful, why not?

9. Throughout the interview we have talked extensively about patient motivation and your role as a PT in facilitating adherence. We know that adherence is often determined by a patient's feelings of autonomy, competence, and connectedness to the task, PT or environment. Do you agree?

a. Please describe the importance of patient autonomy for adherence

b. Please describe the importance of patient competence for adherence

c. Please describe the importance of patient connectedness for adherence

d. Please rank the three in terms of importance for determining patient adherence

10. Do you have anything else to add that might help us understand patient adherence? 